\title{
Impact of Heat Release on Strain Rate Field in Turbulent Premixed Bunsen Flames
}

\author{
Bruno Coriton and Jonathan H. Frank ${ }^{*}$ \\ Combustion Research Facility, Sandia National Laboratories \\ Livermore, California 94551, USA \\ Colloquium on Turbulent Flames \\ * Corresponding Author: Jonathan H. Frank \\ P.O. Box 969, MS. 9053 \\ Livermore, CA 94551, USA \\ Fax: (925) 294-2595 \\ jhfrank@sandia.gov
}

Color reproduction charges will be paid for those figures that in the revision stage cannot be reproduced in black and white without loss of clarity.

\begin{tabular}{|c|c|c|c|c|c|}
\hline \multirow[t]{3}{*}{ references } & \multicolumn{2}{|l|}{19} & & \multirow{3}{*}{$\begin{array}{l}\text { equiv. words } \\
\text { Caption }\end{array}$} & \multirow{3}{*}{\begin{tabular}{|l}
4079 \\
367 \\
Figure total
\end{tabular}} \\
\hline & Figure & & & & \\
\hline & height [mm] & \# columns & equiv. words & & \\
\hline Fig. 1 & 37 & 1 & 104 & 12 & 116 \\
\hline Fig. 2 & 43 & 2 & 232 & 41 & 273 \\
\hline Fig. 3 & 58 & 1 & 151 & 47 & 198 \\
\hline Fig. 4 & 81 & 2 & 402 & 46 & 448 \\
\hline Fig. 5 & 89 & 1 & 218 & 43 & 261 \\
\hline Fig. 6 & 75 & 1 & 187 & 38 & 225 \\
\hline Fig. 7 & 43 & 1 & 116 & 23 & 139 \\
\hline All figures & & & & & 1659 \\
\hline & \#text lines & \#columns & & & \\
\hline Table 1 & 3 & 1 & & & 38 \\
\hline Table 2 & 5 & 1 & & & 53 \\
\hline Total & & & & & 6196 \\
\hline
\end{tabular}




\title{
Impact of Heat Release on Strain Rate Field in Turbulent Premixed Bunsen Flames
}

\author{
Bruno Coriton and Jonathan H. Frank ${ }^{*}$ \\ Combustion Research Facility, Sandia National Laboratories \\ Livermore, California 94551, USA
}

The effects of combustion on the strain rate field are investigated in turbulent premixed $\mathrm{CH}_{4}$ /air Bunsen flames using simultaneous tomographic PIV and $\mathrm{OH}$ LIF imaging measurements. Tomographic PIV provides three-dimensional velocity measurements, from which the complete strain rate tensor is determined. The OH LIF measurements are used to determine the position of the flame surface and the flame-normal orientation within the imaging plane. This combination of diagnostic techniques enables measurements of divergence as well as flame-normal and tangential strain rates, which are otherwise biased using only planar measurements. Measurements are compared in three lean-to-stoichiometric flames that have different amounts of heat release and Damköhler numbers greater than unity. The effects of heat release on the principal strain rates and their alignment relative to the local flame normal are analyzed. The extensive strain rate preferentially aligns with the flame normal in the reaction zone, which has been indicated by previous studies. The strength of this alignment increases with increasing heat release and, as a result, the flame-normal strain rate becomes highly extensive. These effects are associated with the gas expansion normal to the flame 
surface, which is largest for the stoichiometric flame. In the preheat zone, the compressive strain rate has a tendency to align with the flame normal. Away from the flame front, the flame - strain rate alignment is arbitrary in both the reactants and products. The flame-tangential strain rate is on average positive across the flame front, and therefore the turbulent strain rate field contributes to the enhancement of scalar gradients as in passive scalar turbulence. Although increases in heat release result in larger positive values of the divergence as well as flame-normal and tangential strain rates, the tangential strain rate has a weaker dependence on heat release than the flamenormal strain rate and the divergence.

Keywords: tomographic PIV; turbulent premixed flames; strain rate; dilatation.

\section{Introduction}

The strain rate plays an important role in turbulent mixing by enhancing or suppressing scalar gradients. The turbulence-scalar interaction is strongly dependent on the magnitude of the strain rate tensor eigenvalues, also known as principal strain rates, and their alignments with the scalar gradients [1-5]. In non-reacting turbulent flows, scalar gradients are produced by stretching of the scalar isosurfaces. In reacting flows, heat release introduces additional effects such as dilatation and changes in the gas diffusivity, viscosity and density. These changes to the fluid properties can in turn affect the strain rate field and its effect on the production of scalar gradients.

In turbulent premixed combustion, the dynamics of the flame-strain rate interaction involves the alignment of the principal strain rates with the flame normal [1]. The flame-normal and tangential strain rates can be expressed as: $S_{n}=n_{i} n_{j} s_{i j}$ and $S_{t}=\left[\delta_{i j}-n_{i} n_{j}\right] s_{i j}$, where $s_{i j}=\frac{1}{2}\left(u_{i, j}+u_{j, i}\right)$ are the nine 
components of the strain rate tensor and $n=-\nabla c /|\nabla c|$ is the flame-normal unit vector pointing toward the reactants with $c$ representing the instantaneous reaction progress variable. The flame-normal strain rate can be written in terms of the principal strain rates, $s_{i}$, as: $S_{n}=s_{1} \cos ^{2} \theta_{1}+s_{2} \cos ^{2} \theta_{2}+$ $s_{3} \cos ^{2} \theta_{3}$, where $\theta_{i}$ are the angles between the strain rate eigenvectors and flame-normal unit vectors. The principal strain rates satisfy the relation $s_{1} \geq s_{2} \geq s_{3}$. In non-reacting turbulence, $s_{1}+s_{2}+s_{3}=0$ and the maximum and minimum principal strain rates, $\mathrm{s}_{1}$ and $\mathrm{s}_{3}$, correspond to the most extensive and compressive strain rate components, respectively. In reacting flows, the sum of the principal strain rates is non-zero because of dilatation: $\Delta=s_{1}+s_{2}+s_{3}=S_{n}+S_{t}$. The flame-normal and tangential strain rates are determined by dilatation and turbulent strain.

The tangential strain rate, $S_{t}$, is a key quantity in the transport of scalar gradients [1], critical to the modeling of turbulent premixed flames in the context of Reynolds Averaged Navier-Stokes (RANS) and flamelet-based models for Large-Eddy Simulations (LES) [1,4,6-8]. In passive scalar turbulence, $S_{t}$ is dominantly positive, resulting in the production of scalar gradients. In turbulent flames, an understanding of the turbulent transport of scalar gradients requires analysis of the alignment of the principal strain rates with the flame surface and its relation to heat release.

DNS studies have contributed to our understanding of the strain rate field in turbulent premixed flames. In particular, Swaminathan and Grout [1] established the preferential alignment of the extensive strain rate eigenvectors with the flame normal unit vector, which is a different behavior from that of passive scalar gradients. In moderately turbulent flames, scalar gradients, strain rate and dilatation are strongly correlated with flame surface curvature $[2,3]$. However, the preferential alignment of the most extensive strain rate with the flame normal seems to be significantly reduced as the Damköhler number decreases $[4,5]$.

A few experimental studies on turbulent premixed flames [9-11] have investigated the preferential alignment of the strain rate eigenvectors with the flame surface, but the velocity 
measurements have been limited to a single plane and therefore unable to assess the three-dimensional orientation of the velocity gradients. In particular, the full orientation of the principal strain axes as well as the divergence and the flame-tangential strain rate cannot be determined from planar PIV measurements. However, cross-plane OH PLIF imaging can provide information on the threedimensional orientation of the flame fronts [11]. Ultimately, the development of three-dimensional imaging capabilities is required to fully capture the dynamics of the turbulence-flame interaction. Tomographic particle image velocimetry (TPIV) is an advanced PIV technique that provides instantaneous measurements of all three velocity components within a three-dimensional probe volume [12]. TPIV enables measurements of the nine components of the velocity gradient tensor from which the complete strain rate tensor and the divergence can be derived. TPIV measurements in flames $[13,14]$ have potential to unveil new insights into turbulent combustion [15,17].

In the present study, we use simultaneous TPIV and OH LIF imaging measurements to analyze the strain rate field in turbulent premixed Bunsen flames. The effects of heat release on the strain rate field statistics are evaluated by comparing measurements in lean-to-stoichiometric flames. The results are presented in the following three parts: 1) strain rate properties of the comparable non-reacting turbulent flow, 2) strain rate-flame alignment and 3) variations of the strain rates across the flame fronts.

\section{Experimental Methods}

\subsection{Flow Conditions}

Measurements were performed in turbulent premixed $\mathrm{CH}_{4} /$ air flames stabilized on a piloted Bunsen burner of nozzle diameter $36 \mathrm{~mm}$, at a distance of $\mathrm{Y}=50 \mathrm{~mm}$ above the nozzle exit where the influence of the pilot was reduced [18]. An inlet flow rate of 450 LPM was used for the three flames. Turbulence was generated by a combination of two perforated plates housed inside the nozzle. Table 1 lists a series 
of relevant turbulence properties for a non-reacting jet of stoichiometric reactants measured using $10 \mathrm{kHz}$ TPIV at the same location of measurements in the flames, including the mean and RMS axial velocities, $U$ and $u^{\prime}$, respectively, the integral lengthscale, $l^{\prime}$, estimated based on the time-correlation of the streamwise velocity component, the turbulent Reynolds number, $R e_{t}=u^{\prime} l^{\prime} / v$, the Kolmogorov lengthscale, $\eta \propto l^{\prime} R e_{t}^{-0.75}$, and the average strain rate tensor norm, $\langle|s|\rangle$. Three lean-to-stoichiometric premixed flames of equivalence ratios $\varphi=0.65,0.80$ and 1.00 were compared (Table 2), corresponding to increasing heat release parameters, $\tau=\left(T_{b}-T_{u}\right) / T_{u}$, decreasing $u^{\prime} / S_{L}$ velocity ratios and turbulent Damköhler numbers, $D a_{t}=\left(l^{\prime} / u^{\prime}\right) /\left(l_{F} / S_{L}\right)$, greater than unity, with the reactant and product temperatures labelled $T_{u}$ and $T_{b}$, respectively, the unstrained laminar flame speed, $S_{L}$, and flame thickness, $l_{F}$. Thus, the turbulent flames nominally belong to the flamelet regime of turbulent premixed combustion.

\begin{tabular}{cccccc}
\hline $\boldsymbol{u}^{\prime}$ & $\boldsymbol{u}^{\prime} / \boldsymbol{U}$ & $\begin{array}{c}\boldsymbol{l}^{\prime} \\
(\mathbf{m m})\end{array}$ & $\begin{array}{c}\boldsymbol{\eta} \\
(\boldsymbol{m m})\end{array}$ & $\boldsymbol{R} \boldsymbol{e}_{\boldsymbol{t}}$ & $\begin{array}{c}\langle|\boldsymbol{s}|\rangle \\
(\mathbf{m} / \mathbf{s})\end{array}$ \\
\hline 0.62 & $8.2 \%$ & 5.0 & 0.08 & 250 & 535
\end{tabular}

Table 1 - Non-reacting flow field properties

\begin{tabular}{ccccc}
\hline $\boldsymbol{\varphi}$ & $\boldsymbol{\tau}$ & $\begin{array}{c}\boldsymbol{S}_{\boldsymbol{L}} \\
(\boldsymbol{m} / \boldsymbol{s})\end{array}$ & $\boldsymbol{u}^{\prime} / \boldsymbol{S}_{\boldsymbol{L}}$ & $\boldsymbol{D a}_{\boldsymbol{t}}$ \\
\hline 0.65 & 4.8 & 0.15 & 4.1 & 1.7 \\
0.80 & 5.8 & 0.24 & 2.6 & 3.0 \\
1.00 & 6.5 & 0.34 & 1.8 & 5.1 \\
\hline
\end{tabular}

Table 2 - Turbulent premixed flame properties

\subsection{Experimental Apparatus}

The measurements were performed using a high repetition rate imaging system that is described elsewhere [14]. The focus of the present study is the analysis of the three-dimensional flow field 
structure of turbulent flames rather than their temporal evolution. The experimental setup for simultaneous TPIV and OH PLIF measurements is depicted in Fig. 1. TPIV measurements were performed at a repetition rate of $10 \mathrm{kHz}$ in a probe volume of dimensions $20.7 \times 13.9 \times 1.13 \mathrm{~mm}^{3}$, containing $202 \times 136 \times 12$ vectors with $103 \mu \mathrm{m}$ spacing. The TPIV system consisted of a high speed, diode-pumped dual-head Nd:YAG laser and a set of four high speed CMOS cameras. The cameras were positioned on the same side of the laser beam at angles of \pm 15 and \pm 34 degrees with respect to the Zaxis and were operated at $20 \mathrm{kHz}$ in a frame straddling mode using a $1024 \times 768 \mathrm{px}^{2}$ region of the detector with a projected pixel size of approximately $18 \mu \mathrm{m} / \mathrm{px}$. As detailed in Ref. [14], computation of the velocity vectors consisted of: 1) preprocessing the raw particle images, 2) reconstructing the particle volume distribution, 3) calculating the velocity vectors by iterative volume cross-correlation for a final volume interrogation size of $(24)^{3} \mathrm{vx}^{3}=(413)^{3} \mu \mathrm{m}^{3}$ with $75 \%$ overlap and 4) removing/replacing spurious vectors and reducing the measurement noise with a spatial filter. The smallest turbulence length scale that could be resolved, which according to the Nyquist criterion corresponds to twice the dimension of the interrogation region, was $826 \mu \mathrm{m} \approx 10 \eta \approx l^{\prime} / 6$. The accuracy of the three-dimensional velocity measurements and their gradients was evaluated in previous studies [14-15].

OH LIF imaging measurements were performed simultaneously at $10 \mathrm{kHz}$ to determine the flame front contours in each TPIV snapshot. The second harmonic of a $10 \mathrm{kHz}$ Nd:YAG-pumped dye laser ( $90 \mu \mathrm{J} /$ pulse) was tuned to a wavelength of $283.3 \mathrm{~nm}$ to excite the $\mathrm{Q}_{1}(7)$ transition of the A-X $(1,0)$ band of $\mathrm{OH}$. The OH-LIF signal was recorded using a high-speed intensified CMOS camera with a spatial resolution of approximately $100 \mu \mathrm{m} / \mathrm{px}$. Further details are available in Ref. [14]. 


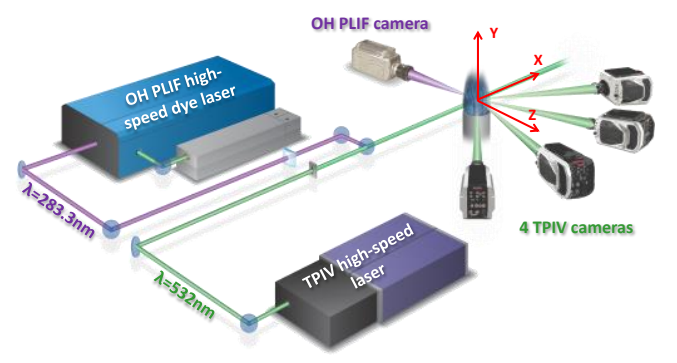

Figure 1 - Schematic of experimental setup for simultaneous TPIV measurements and OH LIF imaging.

\section{Results and Discussion}

The effect of heat release on the strain rate field was investigated by comparing the measurements in the three lean-to-stoichiometric premixed flames of Table 2. The fluid dynamic properties of the three jets were identical and, therefore, differences in the strain rate field could be attributed to the flame properties. The statistical analysis of each flame was performed using 5000 simultaneous TPIV and OH LIF measurements from a $500 \mathrm{~ms}$ time sequence. Properties of the strain rate field in the non-reacting turbulent flow are analyzed first.

\subsection{Non-reacting turbulence}

In order to discern the interactions of combustion with the strain rate field, we first consider the strain rate field for the corresponding isothermal flow with the conditions in Table 1. The turbulent jet consisted of a non-reacting, stoichiometric $\mathrm{CH}_{4} /$ air mixture. PDFs of the principal strain rates, $s_{i}$, are presented in Fig. 2a. The principal strain rates conform to the relationships $s_{1} \geq s_{2} \geq s_{3}$ and $s_{1}+s_{2}+$ $s_{3}=0$, such that the most extensive and compressive strain rates are positive and negative, respectively. The sign of $s_{2}$ can be either positive or negative, implying that the local intermediate principal strain rate can be either extensive or compressive. The distribution of $s_{2}$ is slightly skewed toward positive values with a small mean value of 40/s, in agreement with previous studies of non-reacting turbulence [16]. 
In order to determine the interaction between the flame and the strain field, we must first evaluate whether the principal strain rates in the isothermal jet have a preferential orientation that could influence the results in the flames. The alignments of the strain rate eigenvectors with respect to the burner axes in the non-reacting flow are presented in Figs. $2 b$ and $2 c$. The three-dimensional eigenvector orientation is defined with respect to the coordinate system shown in Fig. 1 by the angles $\theta_{x y}$ and $\theta_{z}$, where $\theta_{x y}$ is the angle of the eigenvector projection in the XY-plane with respect to the positive $\mathrm{X}$-axis and $\theta_{z}$ is the angle of the eigenvector with respect to the Z-axis. PDFs of the angular distributions are shown using $\cos \theta_{z}$ and $\theta_{x y}$. The use of $\cos \theta_{z}$ instead of $\theta_{z}$ eliminates the inherent geometrical bias in spherical coordinates such that a flat PDF of $\cos \theta_{z}$ indicates a random angular distribution of the eigenvector. For the intermediate principal strain rate, $s_{2}$, Fig. $2 \mathrm{~b}$ shows that the probability of $\cos \theta_{z}=1$ is approximately five times larger than the probability of $\cos \theta_{z}=0$. Therefore, $s_{2}$ is preferentially aligned with the Z-axis, which is consistent with the mean flow field in this axisymmetric burner. As another measure of the strength of the preferential orientation of $s_{2}$, we use the PDF to calculate the probability that $\theta_{z}$ is less than 45 degrees. This probability is however only $50 \%$ for this flow field, corresponding to a relatively weak alignment. Figure $2 \mathrm{~b}$ also shows that the eigenvectors $s_{1}$ and $s_{3}$ have a peak near $\cos \theta_{z}=0$, indicating a tendency of $s_{1}$ and $s_{3}$ to be oriented parallel to the XY-plane, and this tendency is slightly stronger for $s_{1}$. In Fig. 2c, all of the PDFs of $\theta_{x y}$ are uniform, signifying that the eigenvector projections in the $\mathrm{XY}$-plane do not have any preferential orientation with respect to the $\mathrm{X}$ and $\mathrm{Y}$-axes. Overall, these results indicate that the strain rate eigenvectors in the non-reacting flow do not have a very strong preferential orientation with respect to the burner axes. 

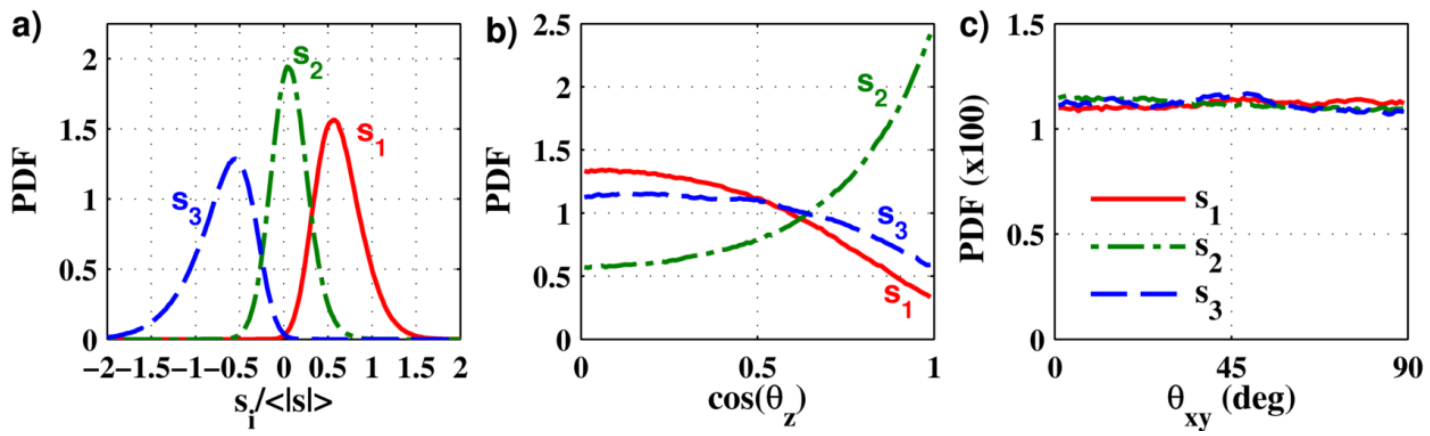

Figure 2 - a) PDFs of the principal strain rates normalized by the mean strain rate tensor norm, $\langle|s|\rangle=535 \mathrm{~s}^{-1}$ (Table 1), b) PDFs of the angle of the principal strain rate axes with respect to the $Z$-axis and c) with respect to the $X$-axis in the $X Y$ plane.

\subsection{Flame-normal alignment of the strain rate eigenvectors}

The effects of the turbulent strain rate on the dissipation rate of the progress variable are strongly dependent on the alignment of the principal strain rates with respect to the flame surface. To evaluate statistics of the flame-strain rate alignment, we determined the flame-normal unit vectors using the $\mathrm{OH}$ LIF images. OH LIF measurements provide a useful marker of the premixed flame front [9-11, 17-18] since the rapid rise of the $\mathrm{OH}$ concentration in the reaction zone can be detected in most turbulent premixed flames. Figure 3 shows a single-shot OH LIF measurement overlapped with the projection of the extensive strain rate eigenvectors in the $\mathrm{OH}$ LIF imaging plane (XY-plane). The flame front contour was identified as the locations of the peak magnitude of gradients of the OH LIF signal, as described in Ref. [19]. The flame-normal unit vectors, $n$, were computed at positions along the flame contour. The magnitudes and orientations of the principal strain axes were then determined along the flame-normal coordinate, $x_{n}$, defined as the distance from the flame front with positive and negative values corresponding to the reactants and flame products, respectively. These measurements were sampled along the local flame normal direction extending $3 \mathrm{~mm}$ on each side of the flame front. As a result of flame folding, the sampled regions along the local flame normal could intersect more than one flame 
front, in which case the strain rate data points were only associated with the nearest flame surface in the statistical analysis.

$\mathrm{OH}$ LIF imaging does not provide measurements of the actual progress variable, and therefore the flame-normal unit vector orientation is strictly only valid at the location of the OH LIF layer. However, the flame conditions are within the turbulent premixed flamelet regime at $D a_{t}>1$, and the scalar isosurfaces are expected to remain nearly parallel in the neighborhood of the flame front, thereby enabling this approach to identify the flame normal. Furthermore, flame orientation is only determined in the OH LIF imaging plane, which can introduce a bias [11]. However, symmetry of the mean flow field with respect to the $\mathrm{OH}$ LIF imaging plane reduces the average bias from 3D flame orientation on the flame-conditional statistics.

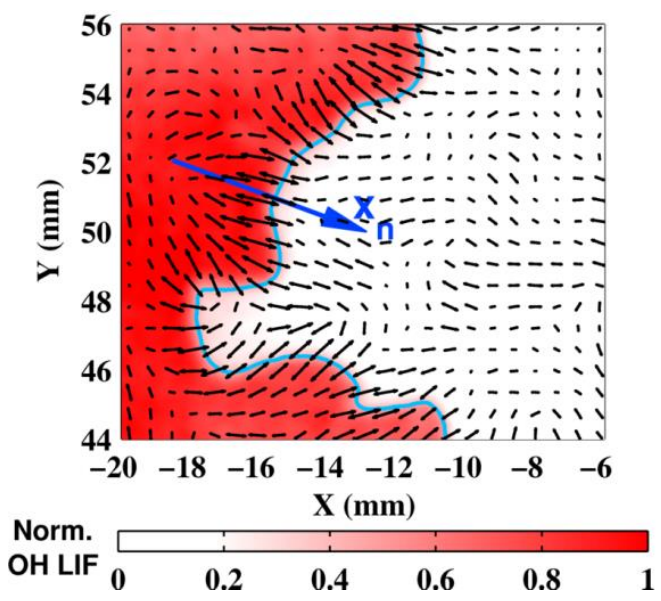

Figure 3 - Single-shot measurement of extensive strain rate eigenvectors ( 1 out of 36 eigenvectors displayed) superimposed on simultaneous OH LIF snapshot. Length of the arrows scaled according to the magnitude of the extensive principal strain rate. Light blue curve indicates the flame front contour, and the dark blue vector is an example of the local flame normal direction.

In Fig. 3, many of the extensive strain rate eigenvectors located near the flame front are nearly orthogonal to the flame front contour, identified in the figure by a blue line corresponding to an isocontour of the normalized OH LIF signal of 0.5 . In contrast, the orientation of the $s_{1}$-eigenvectors is 
more scattered in the reactant and product regions away from the flame front. These observations are reflected in the angular distributions of the principal strain rates with respect to the flame normal at the flame front $\left(x_{n}=0 \mathrm{~mm}\right)$ shown in Fig. $4 \mathrm{a}$. For the three premixed flames, the PDFs of $s_{1}-n$ alignment have a peak at $\cos \theta_{1}=1$, indicating that the $s_{1}$-eigenvectors are preferentially aligned with the flame normal. This alignment is strongest for $\varphi=1.00$ and weakest in the leanest flame for $\varphi=0.65$. The corresponding probabilities that the eigenvector angles with respect to the flame normal are less than 45 degrees are $74 \%$ and $61 \%$ for $\varphi=1.00$ and $\varphi=0.80$, respectively, and only $38 \%$ for $\varphi=0.65$. For $\varphi=1.00$ and $\varphi=0.80$, the PDFs of the eigenvectors of $s_{2}$ and $s_{3}$ have a peak at $\cos \theta_{2}=0$ and $\cos \theta_{3}=0$, respectively, indicating that $s_{2}$ and $s_{3}$-eigenvectors are preferentially oriented orthogonal to the flame normal. For $\varphi=0.65$, however, the distribution of $\cos \theta_{3}$ is more uniform, signifying that the orientation of the $s_{3}$-eigenvectors with respect to the flame surface is more arbitrary.
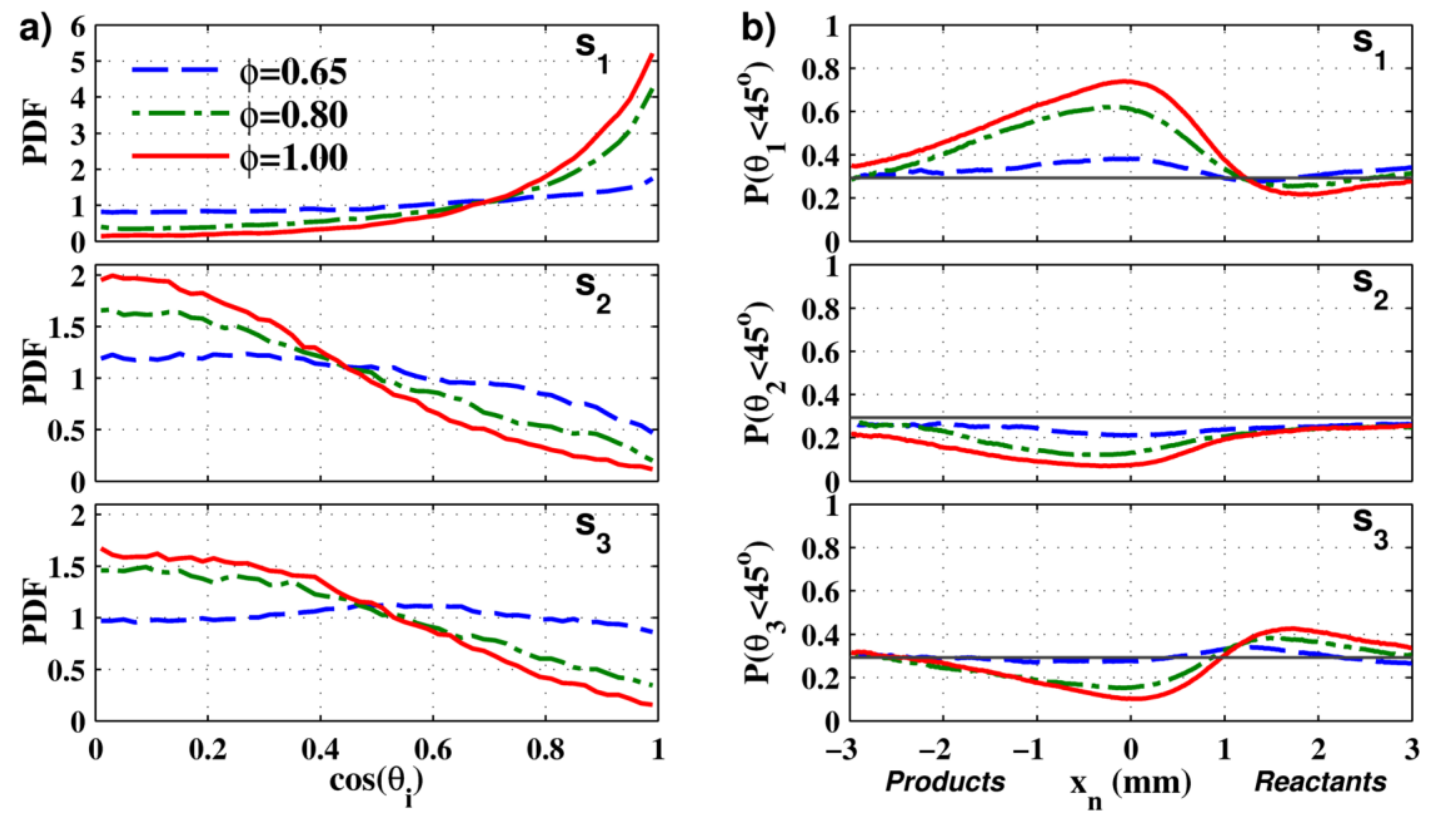

Figure 4 - a) PDFs of strain rate eigenvector alignment with the flame-normal unit vector at the flame front $\left(x_{n}=0\right)$. b) Flame-normal conditional probabilities for $\theta_{i}<45^{\circ}$ as a function of the flame-normal coordinate, $x_{n}$. Black solid line indicates the probability for a random angular distribution. 
The dependence of the flame-strain rate alignments on the distance from the flame front is analyzed in Fig. $4 \mathrm{~b}$ where the probabilities of $\theta_{i}<45^{\circ}$ for the three principal strain rates are plotted as a function of the flame-normal coordinate, $x_{n}$. For reference, the probability of $\mathrm{P}\left(\theta_{i}<45^{\circ}\right)=0.29$ corresponding to a randomly oriented three-dimensional vector field is indicated in the plots of Fig. $4 \mathrm{~b}$. When $\mathrm{P}\left(\theta_{i}<45^{\circ}\right)>0.29$, the $s_{i}$-eigenvectors and the flame-normal vectors are preferentially aligned. For all the flames, Fig. $4 \mathrm{~b}$ shows that the probability $\mathrm{P}\left(\theta_{1}<45^{\circ}\right)$ for the extensive strain rate is largest in the reaction zone near $x_{n}=0 \mathrm{~mm}$ and decreases rapidly toward the reactants and more gradually towards the products. The extent of this preferential orientation is most pronounced for $\varphi=1.00$ and decreases for the lean flames in concert with the decrease in the heat release parameter, $\tau$, and Damköhler number, $D a_{t}$ (Table 2). For $x_{n} \leq-3.0 \mathrm{~mm}$ and $x_{n} \geq 1.0 \mathrm{~mm}$, the distribution $\mathrm{P}\left(\theta_{1}<45^{\circ}\right)$ is comparable to that of a random vector field. For all the flames, $\mathrm{P}\left(\theta_{2}<45^{\circ}\right)$ for the intermediate strain rate is a minimum at the flame front and increases gradually toward reactants and products. However, $\mathrm{P}\left(\theta_{2}<45^{\circ}\right)$ remains systematically less than the random orientation value of $29 \%$, which implies that the $s_{2}$-eigenvectors are preferentially aligned orthogonal to the flame normal vectors. The preferential orientation of the $s_{2}$ eigenvectors with the Z-axis in the non-reacting flow field (cf. section 3.1) may contribute to the orthogonality of the $s_{2}$-eigenvectors with the flame normal, which should be further investigated in different burner geometries. For all the flames, the probability $\mathrm{P}\left(\theta_{3}<45^{\circ}\right)$ for the most compressive strain rate also increases away from the flame front toward both reactants and products but only slightly exceeds $29 \%$ between $x_{n}=1.0 \mathrm{~mm}$ and $3.0 \mathrm{~mm}$. Therefore, the $s_{3}$-eigenvectors have a weak preferential alignment with the flame normal vectors in the reactants just ahead of the flame front, in agreement with previous studies $[6,11]$. This result is consistent with the preferential alignment of the compressive stain rate with scalar gradients in non-reacting turbulent flows. Overall, the probability distributions of the leanest flame exhibit a relatively small deviation from that of a randomly distributed 
vector field, and the flame front has a weak impact on the orientation of the principal strain axes across the $6 \mathrm{~mm}$ region straddling the flame front.

\subsection{Flame-normal conditional statistics of strain rate}

The impact of the preferential strain-rate alignment on flame straining depends on the magnitudes of the principal strain rates in the vicinity of the flame front. To evaluate the contributions of each principal strain rate to the flame-normal strain, $S_{n}$, we analyze flame-normal profiles of the principal strain rates and their projections onto the flame-normal unit vector, $n$, in Fig. 5. The strain rates in the figure are normalized with respect to the mean spatiotemporal value of the strain-rate norm, $\langle|s|\rangle=535 \mathrm{~s}^{-1}$, in the non-reacting flow. For reference, the mean normalized values of the principal strain rates in the nonreacting flow are $\left\langle s_{1}\right\rangle /\langle|s|\rangle=0.63,\left\langle s_{2}\right\rangle /\langle|s|\rangle=0.07$, and $\left\langle s_{3}\right\rangle /\langle|s|\rangle=-0.70$. The profiles of the principal strain rates in Fig. 5 peak in the vicinity of the flame front $\left(x_{n}=0 \mathrm{~mm}\right)$ and decrease in the reactants and products with peak heights varying between the three flames. Dilatation from heat release shifts the principal strain rates at the flame front toward positive values, thereby increasing the extensive strain rate and reducing the magnitude of the compressive strain. The magnitude of the shift for the different flames varies with the heat release parameter (Table 2) and is largest for the stoichiometric flame and smallest for the leanest flame. The increase of $s_{1}$ with increasing heat release is consistent with the greater flow acceleration in the flame normal direction with the peak values of $s_{1}$ occurring slightly towards the product side of the flame front. As the gas expansion across the flame increases, $s_{2}$ becomes increasingly positive and therefore more extensive. The smallest strain rate eigenvalue, $s_{3}$, remains negative on average and therefore compressive in the three flames.

The combined effects of flame-induced changes to the magnitudes of the principal strain rates and the alignment of their axes are determined by the projections of the $s_{1}, \mathrm{~s}_{2}$, and $s_{3}$-eigenvectors onto the flame normal direction, as shown in Fig. 5. A comparison of the respective profiles of the principal 
strain rates and their projections along the flame normal reveals that each principal strain rate has different proportions along the flame-normal and tangential directions, consistent with the alignment statistics shown in Fig. 4. The difference between the respective solid and dashed curves corresponds to the tangential strain component, $S_{t}$, which is parallel to the flame surface. The principal strain $s_{1}$ has the greatest contribution to the flame-normal strain, $S_{n}$, since the magnitude of $s_{1}$ is the most enhanced by dilatation and the $s_{1}$-eigenvetors are preferentially aligned with the flame normal vectors (Fig. 4). The contributions of $s_{2}$ and $s_{3}$ to $S_{n}$ are nearly zero in the three flames because of the misalignment of the $s_{2}$ and $s_{3}$-eigenvectors with the flame normal. Therefore, the dilatation-induced increases in $s_{2}$ and $s_{3}$ primarily contribute to flame-tangential strain, $S_{t}$. The dominantly negative values of $s_{3}$ result in compressive strain along the flame surface and a tendency to reduce the flame surface area. In contrast, $s_{1}$ and $s_{2}$ are dominantly positive, and their contribution to $S_{t}$ tends to increase the flame area.

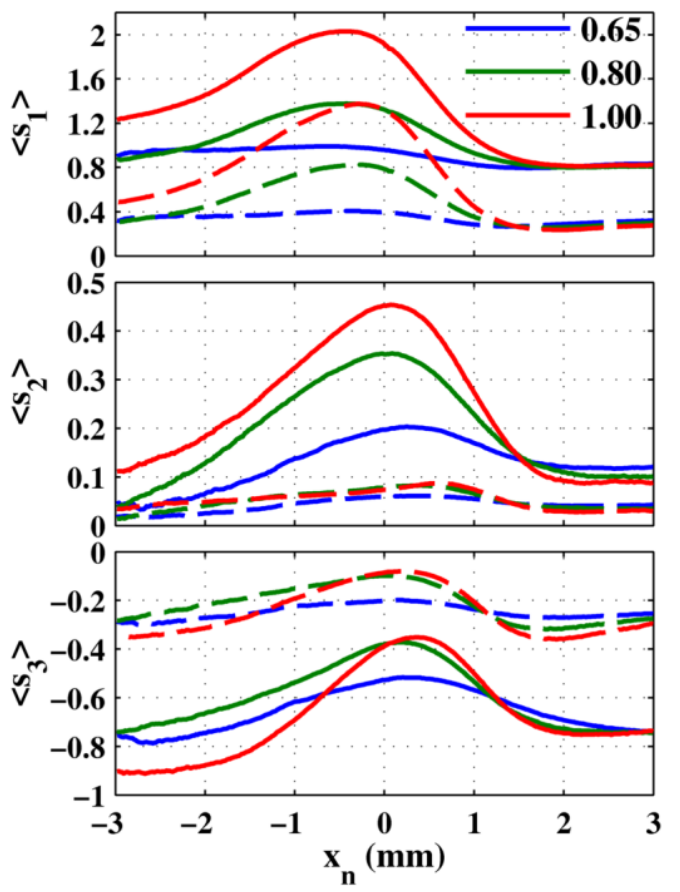

Figure 5 - Flame-normal profiles of mean principal strain rates (solid) and their projections onto the flame-normal direction (dashed) for $\varphi=0.65$ (blue), 0.80 (green) and 1.00 (red). Curves normalized by the mean strain rate norm in nonreacting flow, $\langle|s|\rangle=535 \mathrm{~s}^{-1}$ (Table 1). 
In Fig. 6, we compare the average magnitudes of the flame-normal and tangential stain rates, $S_{n}$ and $S_{t}$, with the divergence, $\Delta$, as a function of the flame-normal coordinate, $x_{n}$. For all the flames, the peak mean divergence is located near $x_{n}=0 \mathrm{~mm}$ with the largest value in the stoichiometric flame and the smallest value in the leanest flame for $\varphi=0.65$. The mean divergence decays to approximately zero in the reactants for $x_{n} \geq 1.5 \mathrm{~mm}$ and more gradually toward the products. The peak average value of $S_{n}$, also located near $x_{n}=0 \mathrm{~mm}$, increases from the lean to stoichiometric flames. The large positive $\left\langle S_{n}\right\rangle$ values near $x_{n}=0 \mathrm{~mm}$ are associated with the gas expansion across the flame front, which induces large extensive strain rates in the flame-normal direction. For $\varphi=0.80$ and $\varphi=1.00,\left\langle S_{n}\right\rangle$ becomes slightly negative for $x_{n} \geq 1.75 \mathrm{~mm}$ as a result of the preferential alignment of the compressive strain rate with the flame normal (Fig. 5). On average, $S_{t}$ is positive across the flame surface with a peak that is shifted towards the reactants relative to the peak of the mean divergence. Positive tangential strain rate contributes to the enhancement of scalar gradients by stretching the flame surface. Although the magnitude of $\left\langle S_{t}\right\rangle$ at $x_{n}=0 \mathrm{~mm}$ increases from the lean to stoichiometric flames, the peak value of $\left\langle S_{t}\right\rangle$ is smaller than that of $\left\langle S_{n}\right\rangle$ for $\varphi=0.80$ and $\varphi=1.00$ but slightly greater than $\left\langle S_{n}\right\rangle$ for $\varphi=0.65$. For the three flames, $S_{t}$ is on average greater than $S_{n}$ in the preheat zone for positive values of $x_{n}$. The crossing location of $S_{t}$ and $S_{n}$ occurs slightly farther into the preheat zone as the heat release increases from the lean to stoichiometric flames. 


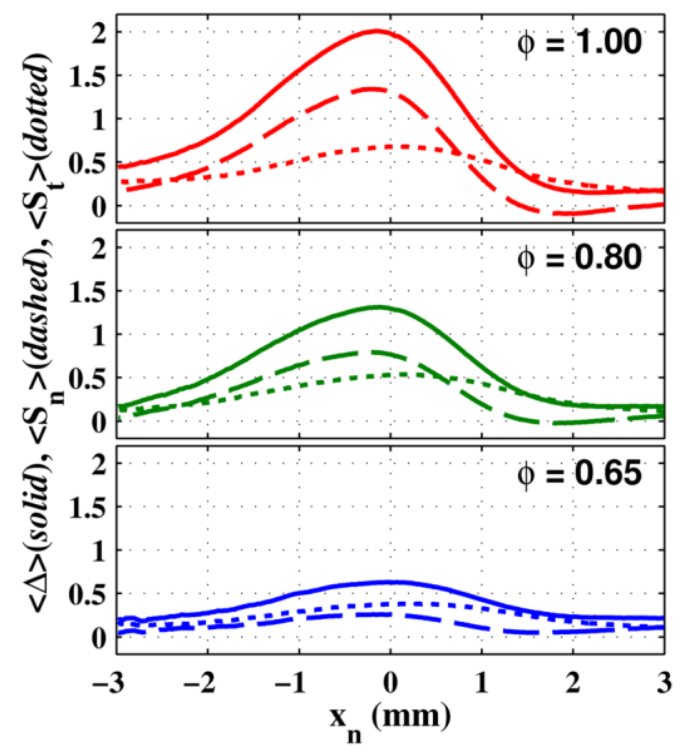

Figure 6 - Flame-normal conditional averages of $\Delta$ (solid), $S_{n}$ (dashed) and $S_{t}$ (dotted) for $\varphi=0.65$ (bottom), 0.80 (middle) and 1.00 (top). Curves normalized by the mean strain rate norm in non-reacting flow, $\langle|s|\rangle=535 \mathrm{~s}^{-1}$ (Table 1).

The results in Fig. 6 demonstrate that $\Delta, S_{n}$, and $S_{t}$ increase with the amount of heat release for the flames considered here. To further understand how gas expansion contributes to flame-normal and tangential strain, we analyze PDFs of the ratio $S_{t} / \Delta$ at $x_{n}=0 \mathrm{~mm}$ in Fig. 7. As the heat release increases from the lean to stoichiometric flames, the PDFs become narrower and their peaks shift from approximately $S_{t} / \Delta=0.6$ for $\varphi=0.65$ to 0.3 for $\varphi=1.00$. This shift to smaller ratios indicates that $\Delta$ increases more rapidly than $S_{t}$ as heat release increases. As heat release increases, flow acceleration normal to the flame also increases, resulting in larger extensive strain, $s_{1}$ (Fig. 5) and a stronger preferential alignment of $s_{1}$ with the flame normal (Fig. 4). Reciprocally, $s_{3}$ becomes less compressive in the flames and preferentially parallel to the flame surface. The distributions of $S_{t} / \Delta$ of the lean flames are broadened by turbulent strain and flame corrugations. In the leanest flame, the broader distribution of $S_{t} / \Delta$ is indicative of the weaker effects of dilatation on turbulence. 


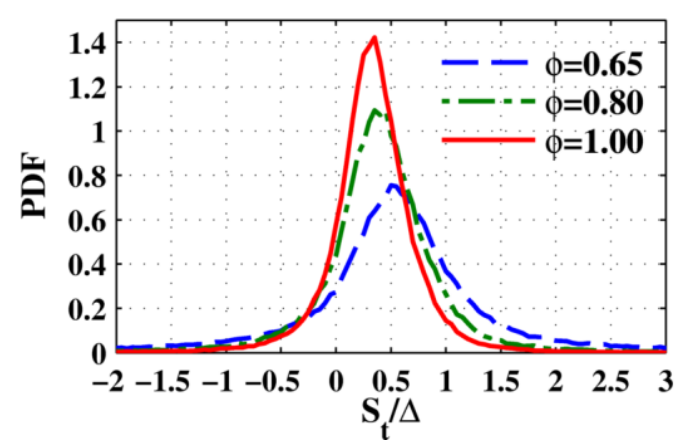

Figure 7 - PDFs of ratio $S_{t} / \Delta$ at the flame front $\left(x_{n}=0 \mathrm{~mm}\right)$ in flames $\varphi=0.65$ (blue), 0.80 (green) and 1.00 (red).

\section{Conclusions}

The effects of heat release on the strain rate field were investigated using simultaneous tomographic PIV and OH LIF imaging measurements. Three-dimensional measurements of the complete strain rate tensor and the divergence enabled the evaluation of the combined effects of strain and dilatation in turbulent premixed flames. Measurements revealed that dilatation can have strong effects on the principal strain rates and their orientations. Along the flame front, the extensive principal strain rate, $s_{1}$, was preferentially aligned with the flame normal, as reported in previous studies. The strength of this alignment increased with increasing heat release. In contrast, the strain rate field in the preheat zone favored the alignment of the compressive strain rate with the flame normal, which was consistent with the alignment in passive scalar mixing. In the products and reactants, the principal strain rates remained more randomly oriented relative to the flame. As dilatation increased, $s_{1}$ and $s_{2}$ became more extensive and $s_{3}$ less compressive. These effects resulted in an elevated flame-normal strain rate at the flame front. The flame-tangential strain rate, $S_{t}$, had a weaker dependence on heat release than the flame-normal strain, $S_{n}$, or the divergence. This effect was associated with gas expansion predominantly normal to the flame surface. The average flame-tangential strain rate was extensive, contributing to the enhancement of scalar mixing by stretching of the flame surface. The coupling between heat release and the strain 
rate field has direct implications for premixed flame modeling since, for instance, the tangential strain rate determines the net effect of dilatation and turbulence-scalar interaction in the transport equations for scalar dissipation and flame surface density.

\section{Acknowledgments}

The authors thank Dr. Hemanth Kolla for insightful discussions and Mr. Erxiong Huang for technical assistance in the laboratory. This research was supported by the U.S. Department of Energy, Office of Basic Energy Sciences, Division of Chemical Sciences, Geosciences, and Biosciences. Sandia National Laboratories is a multiprogram laboratory operated by Sandia Corporation, a Lockheed Martin Company, for the U.S. Department of Energy under contract DE-AC04-94-AL85000. 


\section{References}

1. N. Swaminathan, R.W. Grout, Phys. Fluids 18 (2006) 045102.

2. S.H. Kim, H. Pitsch, Phys. Fluids 19 (2007) 115104.

3. R. Sankaran, E.R. Hawkes, J.H. Chen, T. Lu, C.K., Law, Proc. Combust. Inst. 31 (2007) 12911298.

4. N. Chakraborty, N. Swaminathan, Phys. Fluids 19 (2007)045103.

5. P.E. Hamlington, A.Y. Poludnenko, E.S. Oran, Phys. Fluids 23 (2011) 125111.

6. S. Cant, Turbulent Combustion Modeling, Fluid Mechanics and Its Applications vol. 95, Springer, Netherlands, 2011, p. 63.

7. N. Chakraborty, J.W. Rogerson, N. Swaminathan, Flow, Turbulence Combust. 85 (2010) 25-55.

8. V.K. Chakravarthy, S. Menon, Flow, Turbulence and Combust. 65 (2000) 133-161.

9. G. Hartung, J. Hult, C.F., Kaminski, J.W. Rogerson, N. Swaminathan, Phys. Fluids 20 (2008) 035110.

10. A.M. Steinberg, J.F. Driscoll, N. Swaminathan, Combust. Flame 159 (2012) 2576-2588.

11. T. Sponfeldner, I. Boxx, F. Beyrau, Y. Hardalupas, W. Meier, A.M.K.P. Taylor, Proc. Combust. Inst. 35 (2015) 1269-1276.

12. G.E. Elsinga. B. Wieneke, F. Scarano, B.W. van Oudheusden, Exp. Fluids 41 (2006) 933-947.

13. J. Weinkauff, D. Michaelis, A. Dreizler, B. Böhm, Exp. Fluids 54 (2013) 1624.

14. B. Coriton, A.M. Steinberg, J.H. Frank, Exp. Fluids 55 (2014) 1743.

15. B. Coriton, J.H. Frank, Proc. Combust. Inst. 35 (2015) 1243-1250.

16. W.T. Ashurst, A.R. Kerstein, R.M. Kerr, C.H. Gibson, Phys. Fluids 30 (1987) 2343-2353.

17. A.M. Steinberg, B. Coriton, J.H. Frank, Proc. Combust. Inst. 35 (2015) 1287-1294.

18. J.H. Frank, P.A.M. Kalt, R.W. Bilger, Combust. Flame 116 (1999) 220-232.

19. B. Coriton, J.H. Frank, A. Gomez, Combust. Flame (2016), in press. 